\title{
Figures, Tables, and Boxes
}

\section{Figures}

1.1 Percentage of respondents ranking cyberattacks/cyberthreats as the highest or among the top five biggest concerns to their organizations 6

1.2 Distribution of data breach costs over time (\%) 7

1.3 Most common cyberattacks facing organizations (\%) 13

1.4 A typology of cyberoffenses 16

1.5 Global cybersecurity spending (US\$, billions) 23

1.6 Global cyberinsurance market size (US\$, billions) 24

2.1 GDPR violations and fines imposed in select EU countries (May 25, 2018 to January 27,2020) 36

2.2 China's CS spending (US\$, billions) 45

3.1 The vicious circle of cybercrimes 56

3.2 A comparison of average monthly wages in the US and countries considered to be cybercrime hotspots (US\$) 61

4.1 Number of complaints reported to IC3, 2014-19 73

4.2 Cybercrime conviction rates in select jurisdictions (\%) 75

4.3 Increasing pervasiveness and costs of ransomware 79

5.1 Percentage of countries worldwide with different stages of cybercrime legislation 93

5.2 Percentage of countries worldwide with different stages of data protection and privacy legislation 94

5.3 Percentage of respondents willing to accept less privacy for faster Internet 99

5.4 ICT spending per STEM graduate in some major countries (US\$, thousands) 101 
5.5 Percentage of SMEs facing different types of attacks (2018) 104

6.1 Number of ITU member countries with cybercrime legislation and an NCS (2018) 111

6.2 VC deals and investments in Israeli CS startups 129

6.3 Number of CS companies in Israel at the end of 2018131

7.1 Number of cyberattacks with losses of more than US\$1 million each between 2006 and 2018 attributed to major cyberpowers 134

8.1 Percentage of organizations with different stages of cyberattack and breach response plans 153

8.2 How leaders and average performers differ in key aspects of CS strategy and performance 157

9.1 Most expensive keywords (PPC, US\$, 2017) 179

10.1 Percentage of organizations with different levels of employee CS awareness training programs 189

10.2 Institutional pressures and legitimacy issues in HRM from the CS standpoint 189

10.3 Average salary in North America for CS-related jobs (US\$) 196

10.4 CS-related positions vacant worldwide (millions) 197

11.1 Institutional and technological environment facing SM 216

11.2 Percentage of respondents that ranked different applications as the source of the most malware victimizing their enterprises 218

11.3 Advertising revenue of major SM companies (US\$ billions, 2019) 219

11.4 The top three data exposures by industry/sector (number of records breached, billions, 2019) 220

11.5 Number of unique phishing URLs targeting SM websites (2019 Q4) 223

12.1 Percentage of respondents perceiving different levels of cyberrisk associated with cloud computing 237

12.2 Cloud downtime of major cloud service providers (minutes, early 2015-early 2017) 238

13.1 Percentage of respondents with different meanings of data-minimization policies 252

13.2 Percentage of respondents that considered different factors responsible for driving data-minimization policies 253

13.3 Comparing $4 \mathrm{G}$ and $5 \mathrm{G}$ networks in terms of average throughput per user and latency (MS) 261

14.1 Percentage of respondents perceiving different levels of cyberrisk associated with IoT 265

14.2 Percentage of respondents with different levels of confidence regarding their organizations' IoT devices 266

14.3 Number of IoT devices (billions) 267 
15.1 Percentage of organizations deploying AI in different areas of cybersecurity 285

15.2 The number of devices protected by the safe browsing service (millions) 294

\section{Tables}

1.1 Some examples of cybercrimes associated with various categories of perpetrators and targets 19

1.2 A $2 \times 2$ matrix cyberattacks representing jurisdiction of the target/victim and motivation: An illustration of China 21

1.3 Examples of cyberattacks on power companies and grids 27

2.1 Key principles of FIPs 32

2.2 Examples of some organizations' engagement in deceptive privacy practices and GDPR-related complaints filed against them 39

4.1 Externality mechanisms and feedback systems producing increasing returns in cybercrime-related activities 72

5.1 Some examples of Otherness that make cyberattacks justifiable 97

5.2 A comparison of China, India, Russia, and the US in terms of indicators related to the stock of skills and available opportunities 100

6.1 A comparison of China, Japan, the EU, and the US 127

8.1 Resources in the context of CS: Contrasting practices and perspectives 159

8.2 Strategies for managing vulnerability for various categories of threats and potential consequences 160

8.3 Classification of cyberattacks based on the nature and time variance of threats 162

8.4 Classification of cyberattacks based on the nature of threats and related to actions/positions of the target 163

9.1 Findings of recent surveys regarding the importance placed by consumers on organizations' CS measures (2019) 172

9.2 Some firms facing cyberattacks and the effects on brand reputation 173

9.3 Indicators related to click fraud 174

9.4 Institutional mechanisms associated with criminalizing and stigmatizing click frauds 180

10.1 Some executives who lost jobs due to their failure on the CS front 200

11.1 Facebook-Cambridge Analytica data scandal: A timeline 231

12.1 Organizations' perceptions of the cloud's security: Some representative surveys 235

12.2 Institutional evolution in the cloud industry 239

12.3 A sample of various actors and their actions in shaping cloud-related institutions 244

13.1 Big data characteristics in relation to security, privacy, and welfare concerns 
14.1 Blockchain's potential to address some of the key challenges associated with cloud-based IoT 277

15.1 The evolution of Chronicle: A timeline 295

\section{In Focus Boxes}

1.1 Definitions of Major Terms 8

1.2 Netwalker Ransomware Targets Hospitals during the COVID-19 Pandemic 13

1.3 Healthcare Organizations Faced Cyberattacks during the COVID-19 Pandemic 15

1.4 What Is Cyberinsurance? 25

2.1 The GDPR and Blockchain 34

3.1 Vladimir Zdorovenin Was an Extremely Unlucky Russian Cybercriminal 51

3.2 Mexico's Bandidos Revolutions Team 54

4.1 Evil Corp's Founder Defrauded Victims of Hundreds of Millions of Dollars but Is Living Safely in Russia 75

4.2 Formjacking 77

4.3 Travelex Faces a Ransomware Cyberattack 78

4.4 Sodinokibi Switches to Monero 80

5.1 Romania's Growing CS Prowess 101

5.2 Mondelez International's Lawsuit against Cyberinsurer Zurich 102

6.1 NIST Cybersecurity Framework 114

6.2 China's Measures to Increase Global Influence 122

7.1 Major Adversaries Launched Cyberattacks and Disinformation Campaigns against the US and Its Allies during the COVID-19 Pandemic 135

8.1 Cybersecurity Budgeting 154

8.2 Return on Cybersecurity Investment (ROCSI) 155

9.1 Zoom's Security Debacles Lead to Customer Distrust and Reputation Loss 169

9.2 The FBI Dismantled Click Fraud Operator 3ve 180

10.1 Yahoo's Lack of Priority for CS Led to the Exposure of 3 Billion Accounts 191

10.2 Developing CS Talent among Young People 197

10.3 Employees Need Anti-phishing Training 202

10.4 COVID-19, Remote Working, and CS 203

11.1 The "Facebook Marketing University" in North Macedonia 224

14.1 What Is Blockchain? 275

15.1 Darktrace's Cyber AI Analyst 287

16.1 SolarWinds' Security Weakness Leads to Supply-chain Attacks against Its Customers 301 